\title{
El reconocimiento de los certificados de emisiones reducidas (CER) como un activo intangible
}

\author{
Sonia Milena Gómez Ramírez \\ Diana Marcela Hernández \\ Aura Cristina Ríos Venegas*
}

Gómez Ramírez, S. M., Hernández, D. M. y Ríos Venegas, A. C. (2012). El reconocimiento de los certificados de emisiones reducidas (CER) como un activo intangible. Revista Activos, 18, 149-161.

\section{JEL: Q51}

Recibido: 14 de diciembre de 2012 Aprobado: 5 de enero de 2013

\section{Resumen}

Las conferencias globales para controlar el cambio climático, como la Conferencia de Kioto, han introducido una serie de instrumentos económicos que les permite a las organizaciones convertir el ahorro de emisiones de gases de efecto invernadero en potenciales fuentes de ingreso y financiación para ellas. En Colombia, muchas empresas han entrado en este mercado, pero se presenta gran confusión sobre el tratamiento contable de dichos ingresos. Este trabajo hace una exploración sobre

* Estudiantes de Contaduría Pública de la Universidad Santo Tomás. Una versión preliminar de este trabajo se presentó al Congreso de Estudiantes de Contaduría Pública de la Universidad Santo Tomás en 2012. Correos electrónicos: soniagomez@usantotomas.edu.co, dianajaimes@ usantotomas.edu.co, aurarios@usantotomas.edu.co 
las dinámicas de los certificados de emisión y el tratamiento contable de ellas en el marco regulatorio vigente en Colombia.

\title{
Palabras clave
}

Cambio climático, certificados de emisiones reducidas (CER), reconocimiento contable, activo ambiental.

Gómez Ramírez, S. M., Hernández, D. M. y Ríos Venegas, A. C. (2012). Acknowledging CERs as an intangible asset. Revista Activos, 18, 149-161.

\begin{abstract}
Global conferences to control climate change, such as the Kyoto Conference, have introduced a series of economic instruments that enable organizations to convert the savings of greenhouse gas emissions into potential income sources and funding for them. In Colombia, many companies have joined this market, but there is still great confusion about the accounting treatment of such income. This paper makes an exploration of the dynamics of emission certificates and their accounting treatment within the current regulatory framework in Colombia.
\end{abstract}

\section{Keywords}

Climate change, CER, accounting treatment, environmental asset.

Gómez Ramírez, S. M., Hernández, D. M. y Ríos Venegas, A. C. (2012). La reconnaissance des CER comme immobilisation corporelle. Revista Activos, 18, 149-161.

\section{Résumé}

Les conférences mondiales pour contrôler le changement climatique, comme la Conférence de Kyoto, ont introduit des instruments économiques qui permettent aux organisations de transformer lépargne des émissions de gaz à effet de serre en des sources potentielles de revenus et de financement. En Colombie, beaucoup d'entreprises sont rentrées dans ce marché, mais il y a une grande confusion sur le 
traitement comptable de ses revenus. Ce travail est une exploration des dynamiques des certificats démissions et du traitement comptable de ceux-là dans le cadre réglementaire actuel en Colombie.

\section{Mots-clés}

Changement climatique, CER, reconnaissance comptable, actif environnemental.

\section{Introducción}

Desde 1997 las diferentes organizaciones y gobiernos de los países industrializados firman el Protocolo de Kioto, con el objetivo de proteger el medio ambiente a través de la reducción de emisión de GEI, implementando así mecanismos de flexibilidad dentro de los que se encuentra el mecanismo de desarrollo limpio (MDL) que les permite a los países participantes del protocolo adquirir certificados de emisiones reducidas (CER) provenientes de los países con un bajo nivel de emisión. De hecho, en los últimos años se han negociado en todo el mundo cerca de 421 millones de CER correspondientes a 992 millones de toneladas de carbono, lo que equivale a 18 billones de dólares, que representa, aproximadamente, el 20\% de las transacciones estimadas para este mercado a nivel global.

La presente investigación se centra en el mercado de carbono que consiste en un espacio de compra y venta de certificados de reducción de emisiones de GEI, dada su connotación de transacción susceptible de ser registrada por las empresas, con el fin de reflejar su realidad económica en los estados financieros y así contribuir a la toma de decisiones. Diferentes entidades internacionales han realizado avances sobre el tema, como el International Financial Reporting Interpretations Committee (IFRIC) con su interpretación Draft Interpretation D1 (que meses después fue retirada por el IASB), o el Instituto de Contabilidad y Auditoría de Cuentas en España (ICAC) con la resolución del 8 de febrero de 2006. Sin embargo, la realidad es que no existen parámetros contables que establezcan lo que permita orientar a los profesionales sobre el registro de los CER. Por esta razón, y teniendo en cuenta además que Colombia es uno de los países de Latinoamérica que comercializa CER, se analizará la normatividad nacional e internacional con el fin de identificar por qué dichos certificados cumplen con la definición de activos intangibles 
y su incidencia en la toma de decisiones gerenciales. Asimismo, de qué manera Transmilenio S.A., una de las empresas colombianas que comercializan con CER, refleja en sus estados financieros dichos bonos de carbono.

\section{Generalidades del mercado del carbono y la emisión de CER en Colombia}

Como consecuencia de los cambios climáticos, los países han desarrollado diferentes mecanismos destinados a mitigar los problemas ambientales, producto de las actividades industriales dentro de un mercado competitivo que tiende a la obtención del crecimiento y desarrollo de la economía, dejando de lado externalidades significativas del medio ambiente. Es por ello que en 1997 surge el Protocolo de Kioto como uno de los instrumentos jurídicos internacionales más importantes destinado a luchar contra el cambio climático (Europa. Síntesis de la legislación de la UE, 2010). Por esta razón, se estableció un límite máximo de la cantidad total de emisiones para cada país participante, quien obtiene un monto determinado de permisos de emisión y que puede luego comercializarse en un mercado, donde los participantes que emiten menos de lo permitido pueden vender sus permisos excedentes a aquellos cuyas emisiones exceden su cantidad máxima establecida (Finanzas carbono beta: plataforma sobre financiamiento de carbono para América Latina, s.f.).

Así, quienes pueden reducir sus emisiones sin incurrir en grandes costos tienen el incentivo para hacerlo, pues pueden beneficiarse vendiendo en el mercado sus permisos no utilizados. Por otro lado, para quienes es muy difícil o costoso reducir sus emisiones, la compra de permisos en el mercado puede resultar la opción más eficiente, mediante la financiación de proyectos o abatimiento de estos gases en otras naciones, acreditando tales disminuciones como si hubiesen sido hechas en territorio propio. Los precios de comercialización de estos permisos son determinados en función de la oferta y la demanda de dicho mercado (Cambio climático y mercado del carbono, 2005).

De lo anterior se puede decir que el mercado de carbono es un sistema de comercio a través del cual los gobiernos, empresas o individuos pueden vender o adquirir reducciones de gases de efecto invernadero. 
Según Atehortúa (2007), dentro del mercado del carbono se utilizan tres tipos de mecanismos tendientes a la comercialización de reducción de emisiones:

- Comercio internacional de emisiones (CIE): se da entre países industrializados y se basa en la compra de derechos de emisión a países por debajo de sus cuotas. Las unidades de venta se denominan unidades de cantidades asignadas (UCA).

- Implementación conjunta (IC): está basada en la compra de derechos de emisión resultantes de proyectos implementados conjuntamente entre países industrializados. Las unidades de venta se denominan unidades de reducción de emisiones (URE).

- Mecanismo de desarrollo limpio (MDL): permite la comercialización de reducción de emisiones resultantes de la implementación de proyectos MDL en países en vías de desarrollo. Las unidades de venta se denominan reducciones certificadas de emisiones (RCE o CER) (p. 7).

Los CER han tenido gran impacto y adopción por parte de algunos países interesados en la reducción de los GEI. Estos certificados son emitidos exclusivamente por la Junta Ejecutiva de la ONU mediante bonos que equivalen a una tonelada métrica de dióxido de carbono cada uno, caracterizado por ser único, transferible y negociable, siendo plena y libremente comercializable por sus titulares antes, durante o después de su emisión (Pereyra, 2010).

\section{Proceso de adquisición de CER}

La obtención de los CER se logra mediante un ciclo consecutivo, que se inicia con la recolección de la información básica del proyecto por ejecutar mediante una encuesta relacionada con las técnicas, procedimientos, criterios y su dimensión. Consolidada esta información, se procede a elaborar el documento de diseño del proyecto (PDD), en el que se deben especificar impactos ambientales, localización, contexto y tecnología para el cumplimiento y seguimiento (Guía de desarrollo limpio: una introducción a los mercados de crédito de carbono y mecanismos de desarrollo limpio, CeroCO2 - South Pole - AECID, 2011).

Posteriormente, el documento requiere una aprobación formal de la autoridad nacional designada (DNA) del país anfitrión donde se realizará el proyecto, donde 
la entidad operacional designada (DOE) evaluará las actividades, corroborará si los requerimientos de participación fueron cumplidos y si las provisiones del monitoreo, verificación y reportes cumplen con las reglas del MDL. Esta aprobación tarda entre cuatro y ocho semanas. De igual manera, se tendrá en cuenta si los comentarios de las consultas locales se procesaron e incluyeron durante la validación. Si en el documento se especifican y se cumplen los requerimientos antes mencionados, la DOE enviará el informe de validación y la documentación del proyecto a la UNFCCC (Marco de las Naciones Unidas Sobre el Cambio Climático), solicitando el registro de las actividades del proyecto (CeroCO2, South Pole y AECID, 2011).

Una vez recibida la solicitud de la emisión, el comité ejecutivo publica los CER dentro de los siguientes quince días, así:

- Un 2\% de los CER en un fondo creado especialmente para contribuir para la adaptación al cambio climático de los países en vías de desarrollo.

- Los CER restantes se consignan en las cuentas de registro de las partes y participantes del proyecto (CeroCO2, South Pole y AECID, 2011).

Sobre la emisión de los CER es necesario pagar los correspondientes honorarios a la UNFCCC para cubrir los costos administrativos, equivalentes a un 0,1 US/CER para los primeros $15.000 \mathrm{CER}$ publicados y 0,2US/CER para el volumen restante generado en un año (CeroCO2, South Pole y AECID, 2011).

La valoración inicial de este tipo de títulos se establece en el Banco Mundial y con el gobierno holandés, teniendo en cuenta que son los principales compradores de CER en el mercado del carbono (Eguren, 2004, p. 44).

Durante el periodo de acreditación de la actividad del proyecto MDL, que puede fijarse en un periodo de diez años o uno renovable de acreditación de siete años, el ejecutor del proyecto debe realizar un seguimiento de las reducciones de PDD; de igual manera, se debe enviar un informe de seguimiento e inspección que muestre si se cumplen los requerimientos de los PDD registrados. Si como resultado de este informe se evidencian fallas, se deben recomendar cambios en las metodologías de seguimiento y, finalmente, proveer un informe de verificación (CeroCO2). 


\section{Proceso de emisión de CER en Colombia}

Colombia es un país con bajos porcentajes de emisión de GEI y ha sido pionera del mecanismo de desarrollo limpio (MDL) en América Latina, enfocándose en la comercialización de bonos, representados en certificados de emisiones reducidas (CER) (Conservation International, ICFJ y IFEJ, 2005).

En Colombia, la autoridad nacional designada es el Ministerio de Ambiente y Desarrollo Sostenible, mediante la Oficina Colombiana para la Mitigación del Cambio Climático (OCMCC) que promueve la ejecución de dichos proyectos en distintos sectores, especialmente en aquellos que aporten beneficios sociales a causas como la superación de la pobreza y la erradicación de cultivos ilícitos (Finanzas carbono Project, 2009).

La OCMCC analiza y aprueba los proyectos presentados por las empresas, luego procede a enviarlos a una entidad operacional que se encarga de revisarlos, validarlos y, posteriormente, presentarlos ante la junta ejecutiva del MDL para su registro. Este proceso requiere que la OCMCC vigile las prácticas realizadas para comprobar que efectivamente se está reduciendo la emisión de los GEI y reportar nuevamente a la entidad operacional, quien eleva un informe de verificación ante la junta ejecutiva del MDL. Cumplidas las condiciones, esta junta expide el certificado de reducción de emisiones que se negociará con otros países (Finanzas carbono Project, 2009).

En los años 2007 a 2010, en Colombia se recaudaron ingresos por concepto de venta de bonos de carbono representados en CER por un valor de 91.230 millones de dólares. Esta cifra evidencia que Colombia participa de manera activa en la evolución de MDL (Ministerio de Ambiente y Desarrollo Sostenible).

\section{Los CER y su reconocimiento contable}

El mercado de certificados de emisiones reducidas está creciendo debido a la ejecución de políticas y programas nacionales, sectoriales y corporativos de reducción de emisiones de GEI, lo que permite que actualmente Colombia sea el cuarto país 
de Latinoamérica en número de proyectos MDL registrados ante la ONU y el decimoprimero en el mundo (Ministerio de Ambiente y Desarrollo Sostenible, 2012). Por esta razón, es evidente la necesidad de analizar la normatividad colombiana e internacional con el fin de orientar hacia un apropiado reconocimiento contable de los CER, de tal manera que se refleje la realidad económica de las empresas, su contribución al medio ambiente y al proceso de toma de decisiones gerenciales.

El Marco Conceptual de Estándares Internacionales de Información Financiera y el Decreto 2649 de 1993 establecen como una característica para el reconocimiento de un activo que su utilización puede generar beneficios económicos futuros; en otras palabras, la generación de flujos de efectivo o su equivalente para la entidad. A diferencia del Decreto 2649, el Marco Conceptual indica específicamente que el control de la entidad sobre sus recursos está relacionado con el monitoreo e influencia en las actividades que se desarrollan, de tal manera que se le permita verificar si se ajusta con lo planteado y, en caso negativo, corregir las posibles desviaciones, para que se contribuya a la obtención de los ya mencionados beneficios económicos futuros.

Por otro lado, el Decreto 2649 de 1993 se refiere a activos intangibles como:

Los recursos obtenidos por un ente económico que, careciendo de naturaleza material, implican un derecho o privilegio oponible a terceros, distinto de los derivados de los otros activos, de cuyo ejercicio o explotación pueden obtenerse beneficios económicos en varios periodos determinables. Para reconocer la contribución de los activos intangibles a la generación del ingreso, se deben amortizar de manera sistemática durante su vida útil. Esta se debe determinar tomando el lapso que fuere menor entre el tiempo estimado de su explotación y la duración de su amparo legal o contractual.

La NIC 38 habla de activos intangibles como aquellos recursos identificables, de carácter no monetario y sin apariencia física, controlados por la entidad y de los que se espera obtener beneficios económicos en el futuro. Solo se reconocerá cuando los beneficios económicos fluyan a la entidad y el costo del activo pueda medirse de forma fiable, es decir, aunque los flujos de efectivo sean recibidos en un 
tiempo posterior, la empresa tendrá otros beneficios relacionados con su imagen corporativa (párrafo 8).

Según la Guía de desarrollo limpio (2011), durante la aprobación del proyecto, todas las erogaciones efectuadas por la entidad que decide comercializar los bonos deben ser reconocidas como cargos diferidos, de los cuales se esperan beneficios económicos futuros. Sin embargo, la NIC 38 relacionada con activos intangibles, al respecto establece que en la fase de investigación de un proyecto interno la entidad no puede demostrar que exista un activo intangible que genere beneficios económicos en el futuro; por lo tanto, los desembolsos correspondientes se reconocerán como gastos en el momento en que se produzcan (NIC 38, párrafo 54). En el contexto del mercado de bonos de carbono, el proyecto interno a que hace referencia la NIC 38 se relaciona con la implementación del MDL en el interior de la empresa.

En el momento en que se aprueba el proyecto, la entidad emisora de los bonos podrá realizar el registro contable como un activo intangible, puesto que es un bien inmaterial que genera derechos a la empresa como incentivo a la reducción de gases efecto invernadero o adopción de políticas destinadas al mismo fin, de lo cual se espera obtener beneficios económicos futuros. De igual manera, en las notas a los estados financieros se debería realizar una descripción del proyecto realizado con respecto a la inversión y procedimientos requeridos. El registro de los CER como activos intangibles permite obtener información confiable y verídica de las transacciones económicas de la entidad y, por ende, de la situación real de la organización.

Cuando una empresa emite gases efecto invernadero en menor magnitud a la permitida, esta puede solicitar ante la ONU el permiso de emitir certificados de emisiones reducidas para negociar con otras empresas que requieran más nivel de emisión; esto permite que la empresa controle tales recursos puesto que se tiene el activo como un derecho con el que se negocia libremente o no, o en tal caso mantenerlo para entregarlo cuando se hagan emisiones. Por otro lado, aunque los CER se respalden con un documento utilizado como mecanismo formal para las transacciones de dichos certificados, no significa que los CER tengan apariencia física; por lo tanto, cumplen con la definición de activo intangible. Luego de vendidos 
los respectivos bonos, la entidad procedería a cancelar el activo intangible contra el disponible por el pago recibido.

Hoy en día organizaciones colombianas como Megabus S.A., Metrocali S.A., Incauca S.A., Procuenca y Transmilenio S.A., registran los CER como un ingreso operacional por venta, solo en el momento en que se recibe el dinero por estos bonos. Una razón por la que se registran estos bonos como ingreso se relaciona con los beneficios tributarios para las empresas que despliegan proyectos de desarrollo limpio, consistente en la disminución del porcentaje para el pago de impuesto de renta y ganancia ocasional, como incentivo por cooperar con el desarrollo o mejoramiento de las condiciones medioambientales. Este tratamiento contable se puede evidenciar en la Nota a los estados financieros 17: ingresos operacionales, de Transmilenio S.A., que se muestra a continuación:

Tabla 1. Nota 17. Ingresos operacionales-otros servicios

\begin{tabular}{lc}
\hline Otros servicios-explotación colateral & $\mathbf{2 0 1 1}$ \\
\hline Capacitaciones y visitas & $\$ 41.477$ \\
Asistencia técnica & $\$ 64.500$ \\
Organización de eventos & $\$ 506.987$ \\
Reducción de emisión de gases & $\$ 2.643 .898$ \\
Total & $\$ 3.256 .862$ \\
\hline
\end{tabular}

* Corresponden a los ingresos generados y causados por la venta de certificados de reducción de emisión de gases de 2009 y 2010, expedidos de acuerdo con el contrato de compraventa de reducción de emisiones, con relación al proyecto BRT, Colombia Transmilenio Fase II al IV.

Fuente: Transmilenio S.A.

Este esquema de registro contable pasa por alto las características inherentes a los CER, lo que los hace susceptibles de ser representados y revelados en los estados financieros como activos intangibles, de acuerdo con lo indicado en el marco conceptual, y en el caso colombiano por el mismo Decreto 2649. Según Cañibano, cuando los intangibles no están reflejados en el balance, se generan estimaciones 
sesgadas del valor actual de la empresa y su capacidad para la generación de riqueza en el futuro (Cañibano y Sánchez, 1999, p. 46).

Optando por el reconocimiento inicial de los CER como activos intangibles al cumplir con las condiciones de ser identificables, controlables y generadores de beneficios económicos en el futuro, el reconocimiento contable utilizado actualmente para estos recursos cambia por completo, puesto que ya no se reconocería el ingreso, debido a que en el momento en que este derecho se transfiera a otra empresa el paso a seguir es la sustitución del intangible y el reconocimiento del disponible recibido como contraprestación por la venta de tales títulos.

\section{Conclusiones}

En Colombia se han desarrollado mecanismos para apoyar el protocolo de Kioto y así contribuir a mitigar los efectos de la contaminación sobre el medio ambiente, recurriendo a los certificados de emisiones reducidas CER, que luego se pueden comercializar con los diferentes países que necesiten permisos para emitir GEI. Desde el punto de vista contable, el problema surge con el equivocado tratamiento que las empresas emisoras colombianas les dan a estos certificados, debido a que no existen normas específicas que regulen y orienten su revelación y, por ende, son reconocidos frecuentemente como un ingreso operacional producto de su venta. Según el análisis de la normatividad colombiana y las normas internacionales de contabilidad, los CER cumplen con las características fundamentales de los activos intangibles: identificable, sin apariencia física y generadores de beneficios económicos en el futuro.

Dado que los CER cumplen con la definición de activos intangibles, el registro y revelación contable debe cambiar, puesto que no cumple con el principio de devengo o causación que establece que los hechos económicos deben ser reconocidos en el periodo en que se realicen y no solamente cuando sea recibido o pagado el efectivo o su equivalente (Decreto 2649 de 1993). Como consecuencia, la información no es fidedigna sobre todos los hechos y transacciones útiles para los usuarios en el momento de la toma de decisiones, tanto económicas como de gestión ambiental; además, se desconocen las diferentes ventajas competitivas relacionadas con el aumento del valor de las empresas. 
Debido a que en la actualidad se están implementando mecanismos sociales, políticos y económicos para la mitigación de los efectos negativos en el ambiente, y en aras de la convergencia, es necesario establecer normas contables específicas con el fin de orientar a su debido registro contable.

\section{Referencias}

Atehortúa, E. (2007). El mercado de carbono, un nuevo escenario para los negocios internacionales. Recuperado el 1 de abril de 2012, de http://web.usbmed. edu.co/usbmed/mercatura/nro9/elmercadodelcarbono.htm

Cañibano, L., García, M., y Sánchez, P. (1999). La relevancia de los intangibles para la valoración y la gestión de empresas. Revista Española de Financiación y Contabilidad, extraordinario(100), 17(25), 30-32.

Convención de las Naciones Unidas para el Cambio Climático (2011). Guía de desarrollo limpio: una introducción a los mercados de crédito de carbono y mecanismos de desarrollo limpio.

Eguren C., L. (2004). El mercado de carbono en América Latina y el Caribe: balance y perspectivas. Cepal, N.U. (Ed.). Division de Desarrollo Sostenible y Asentamientos Humanos, (83), 29-37.

Giner Inchausti, B. (2007). La contabilidad de los derechos de emisión: una perspectiva internacional. Revista Española de Financiación y Contabilidad, 36(133), 175-193. Madrid, España.

\section{Páginas web consultadas}

Finanzas carbono beta: plataforma sobre financiamiento de carbono para América Latina (s.f.). Recuperado el 4 de abril de 2012, de http://finanzascarbono. org/finanzas-carbono/acerca/comercio-emisiones/ 
Instituto de Estrategias Globales para el Medio Ambiente (s.f.). Recuperado el 15 de marzo de 2012, de http://finanzascarbono.org/comunidad/pg/map_tool/ map/countries

Instituto Nacional de Ecología (2005). Cambio climático en México. Recuperado el 2 de abril de 2012, de http://cambio_climatico.ine.gob.mx/sectprivcc/ mercadobonoscarbono.html

Ministerio de Ambiente y Desarrollo Sostenible (s.f.). Portafolio de MDL en Colombia. Recuperado el 25 de marzo de 2012, de http://www.minambiente. gov.co/contenido/contenido.aspx? catID $=829 \&$ conID $=3046$

Patiño Orozco, E. (2005). Biodiversity Reporting. Recuperado el 1 de abril de 2012, de http://www.biodiversityreporting.org/article.sub?docId=1326 9\&c=Colombia\&cRef $=$ Colombia\&year $=2005 \&$ date $=$ February $\% 202005$

Transmilenio (2003). Recuperado el 26 de marzo de 2012, de http://www.caf.com/ view/project_popup.asp?id=20604\&ms=12

\section{Normas y estándares}

Consejo de Estándares Internacionales de Contabilidad - IASB. Norma Internacional de Contabilidad 38 (2009). Activos intangibles.

Presidencia de la República (1993). Decreto 2649. Por el cual se reglamenta la contabilidad en general y se expiden los principios o normas de contabilidad generalmente aceptados. 